\title{
The Evolution from Digital Mock-Up to Digital Twin
}

\author{
Sheng DAI ${ }^{\mathrm{a}}$, Gang $\mathrm{ZHAO}^{\mathrm{a}}$, Yong $\mathrm{YU}^{\mathrm{a}, \mathrm{b}, 1}$ and Qiangwei BAO ${ }^{\mathrm{a}}$ \\ ${ }^{a}$ School of Mechanical Engineering and Automation, Beihang University, Beijing \\ 100191, PR China \\ ${ }^{\mathrm{b}}$ Beijing Engineering Technological Research Center of High-Efficient \& Green CNC \\ Machining Process, Beijing 100191, PR China
}

\begin{abstract}
This paper summarizes the development and evolution from digital mock-up (DMU) to digital twin (DT) by clarifying the connotation of DT from prospective of digital product definition (DPD). Firstly, taking Airbus as example, the evolution of DMU is introduced, with the detailed analysis of configured DMU, functional DMU, and industrial DMU. Secondly, based on the literature review of DT, the definition, purpose and several applications of DT concept are clearly expounded. Finally, the augmentation for DT and DPD's relationship are deduced.
\end{abstract}

Keywords. digital product definition, digital mock-up, digital twin

\section{Introduction}

Since 1950s, the digital manufacturing concept has emerged with the rapid development of various supporting technologies. Digital manufacturing is an integrated computer system supported by simulation tools, three-dimensional visualization tools, and various collaborative tools, which realizes the DPD and process definition simultaneously. It can be seen that DPD provides the data foundation for digital manufacturing. To achieve fullelement and full-process digital manufacturing, the DPD have to be done accurately and precisely.

Based on the motivation above, the digital manufacturing concept has experienced a development from two-dimensional to three-dimensional. The industry has proposed DMU, virtual prototypes (VP) and other concepts that focus on product design, leading to the improvement of the definition and expression of product information.

Recently, the experience of domestic and foreign manufacturing industries has shown that the product model defined by three-dimensional digitalization has matured and its benefits have been verified repeatedly. However, there are still some challenges in digital manufacturing:

- From the point of view of its connotation, DPD mainly focuses on the designing information and always ignores the data of manufacturing, operation, and main-

\footnotetext{
${ }^{1}$ Corresponding Author: Yong Yu, School of Mechanical Engineering \& Automation, Beihang University, Beijing, PR China; E-mail: yuyong@buaa.edu.cn.
} 
tenance stages. As a result, there is a gap in terms of representing middle- and end-of-product lifecycle-related semantics [1].

- There is a lack of connection between product definition and process definition. For example, DMU defines the assembly relationship of parts/components, but it cannot accurately describe the assembly process.

- The definition of product information is the "idealized description" given by the CAD (computer-aided design) software. Thus, the simulation based on the "idealized description" has limited guidance for real physical objects.

All in all, to meet the needs of intelligent manufacturing and mirror the actual status of the physical objects, the digital twin concept was introduced by Grieves in 2011 [2]. It is suggested that DT can reflect the dynamic mapping of physical product and its digital counterpart and it is the technical core of cyber-physical system (CPS) [3].

The remainder of this work is organized as follows: Section 2 reviews the literature in the evolution of DMU concept. The connotation of DT concept is presented in Section 3 with detailed discussions. The relationship between DT and DPD is discussed in Section 4. Finally, discussion is provided in Sections 5.

\section{The evolution of DMU in Airbus}

With the development of the CAD software, the connotation of DMU has undergone great changes. Take Airbus company as an example, DMU has gradually evolved into CDMU (configured DMU), FDMU (functional DMU) and iDMU (industrial DMU), as shown in Figure 1.

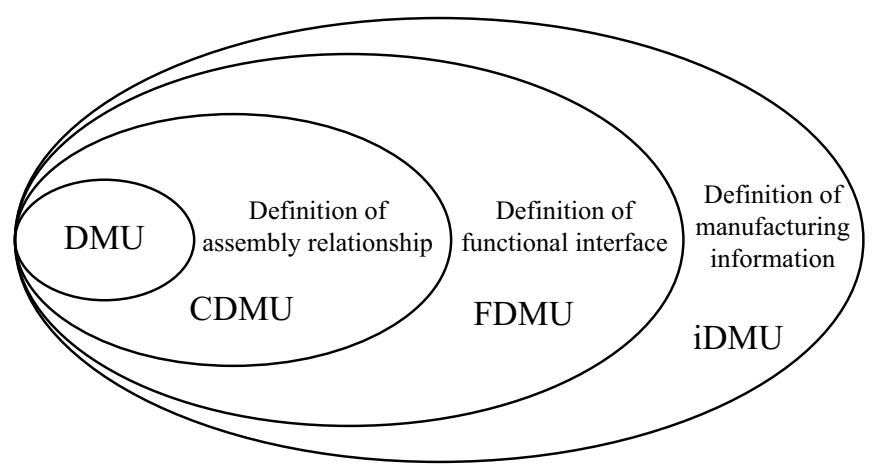

Figure 1. The evolution of DMU in Airbus.

It is believed that a complete DMU should be composed of a three-dimensional model, product structure and product attributes in Airbus. The product structure describes the hierarchical dependence and organizational relationship of the digital model, and the attributes describe the state. This data organization is called configured DMU (configurable DMU) [4], as illustrated in Figure 2.

On the other hand, in order to enrich the functions and behaviors of the DMU in the designing stage, Airbus proposed the functional DMU [5]. FDMU uses the DMU as a carrier to extract components and support requirements and functional analysis, and com- 


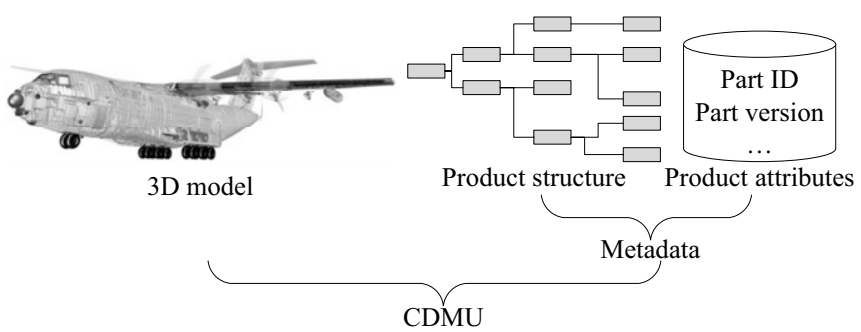

Figure 2. The CDMU concept.

prehensive simulation. The emergence of the FMI standard has profoundly affected the research of FDMU. The FMI standard was first proposed by Daimler AG. The ITI company developed the SimulationX software based on the FMI standard. Dassault Systèmes acquired Dymola software that supports FMI standard.

In order to explore the usage of DMU and optimize the manufacturing process, Airbus implemented the collaborative engineering and iDMU concept in the development of A320neo [6]. In the engineering phase, Airbus reintegrated the functional designing and process manufacturing, and focuses on the definition of products, processes and manufacturing resources from the conceptual stage to mass production. The delivery at this stage were called iDMU. In the manufacturing phase, real data such as the deviation were integrated into iDMU. As shown in Figure 3, when the manufacturing works ends, the output is called as-built iDMU, it is a complete definition of the as-built product.

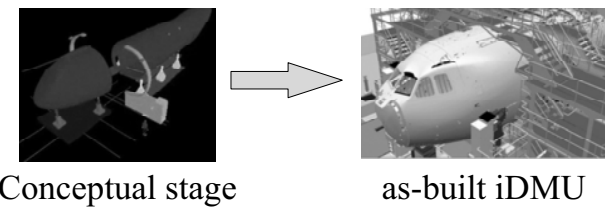

Figure 3. The iDMU concept.

As a result, An iDMU gathers all the product, processes and resources information to model a virtual assembly line and provides a single platform to define and validate the assembly line industrial design [7]. From the perspective of reflecting the physical objects, the as-built iDMU concept is similar with the DT concept. Unfortunately, since it appeared in the research report in 2013, the detailed method of iDMU is still unclear as a commercial secret.

\section{From DMU to DT}

As mentioned above, although the DMU concept in digital manufacturing has been broadened to express more and more information, it still ignores the data of manufacturing, operation, and maintenance stages. But DT technology is considered to be able to describe physical products and effectively manage the life-cycle information. Thus, the DT concept has gradually attracted the attention of academia and industry. 


\subsection{The proposal of DT concept}

As illustrated in Figure 4, the original assumption of DT was introduced by Dr. Michael Grieves in 2002, which was named "conceptual ideal for PLM (product life management) information mirroring". Subsequently, "Mirrored Space Model" and "Information Mirroring Model" had been used to redefine the concept. Finally, the "Digital Twin" was introduced by Grieves and his partner in 2011.

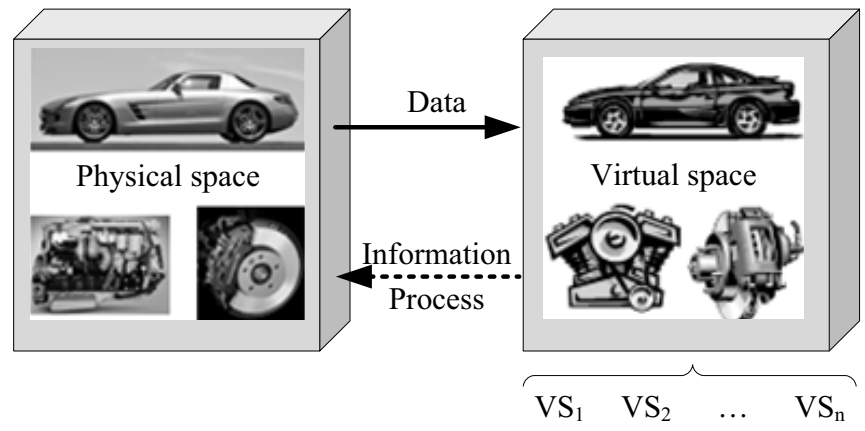

Figure 4. The conceptual ideal for PLM.

Later in 2011, United States Air Force research laboratory adopted the DT concept as a new way to predict the aircraft structural life. In 2012, National Aeronautics and Space Administration defined DT as "an integrated multi-physics, multi-scale, probabilistic simulation of a vehicle or system that uses the best available physical models, sensor updates, fleet history, and so forth, to mirror the life of its flying twin" [8].

\subsection{The definition of DT concept}

After several years, different definitions and explanation of DT came out. For example, DT was seen as the next generation of simulation [9]. Grieves redefined DT as "a set of virtual information constructs that fully describes a potential or actual physical manufactured product from the micro atomic level to the macro geometrical level and any information that could be obtained from inspecting a physical manufactured product can be obtained from its digital twin" [10].

Recently, DT refers to a virtual representation of manufacturing elements, a living model that continuously updates and changes as the physical counterpart changes in a synchronous manner [11]. The role of DT in digital manufacturing is to allow a physical "thing" to be understood for computers and machines by reflecting its physical status.

\subsection{Applications of DT in digital manufacturing}

Since the DT concept was proposed, it has been applied in many industrial fields. The possibility of DT-driven product manufacturing is proposed with an example about the drive shaft machining process [12]. A DT-based manufacturing execution system is developed and validated, combining MTConnect data with production data collected from operators [13]. An approach to modeling product DTs, process DTs, and operation DTs using Automation Markup Language is proposed [14]. A DT-based process evaluation 
method is developed, including the real-time mapping mechanism, the construction of DT-based machining process evaluation framework, and the process evaluation [15]. A generic architecture for cloud-based manufacturing equipment based on DT and big data analysis is illustrated [16]. A novel DT-driven approach for rapid reconfiguration of automated manufacturing systems was proposed and key enabling techniques, including how to twin cyber and physical system and how to quickly bilevel program the production capacity and functionality of manufacturing systems to adapt rapid changes of products were detailed [17]. Zheng et al. introduced a generic CPS system architecture for DT establishment in smart manufacturing with a novel tri-model-based approach for product-level DT development and then conducted a case study of an open source 3D printer DT establishment [18]. Based on the discrete event system modeling theory, a virtual modeling method at a conceptual level and the implementation mechanisms of the virtual-physical connections in practice for establishing DT shop floor was given [19]. Zhuang et al. proposed a DT-based assembly data management and process traceability approach for complex products and the DT-based Assembly Process Management and Control System (DT-APMCS) was designed to verify the efficiency of the proposed approach [20].

In summary, DT concept can be applied to many fields of digital manufacturing, reflecting real conditions, accumulating actual data, paying attention to previously ignored information, mining hidden new knowledge, and optimizing the entire stage of digital manufacturing.

\section{DT and DPD}

Normally, DPD is a process that allows the design team to input all their information into the $3 \mathrm{D}$ model, thus eliminating the need to create a $2 \mathrm{D}$ drawing. For example, DMU involves making the 3D CAD product model the authority dataset, only including the 3D Model and additional information such as parts lists, part coordination documents, etc, within the $3 \mathrm{D}$ viewing area of the model.

On the other hand, with the development of PLM technology, DPD focuses on the research on the unified and consistent definition of product information in different stages. PLM is proposed to manage all the product life-cycle data, and it contains all the contents of PDM (product data management). It supports related activities including designing, manufacturing, sales, and maintenance and effectively manages the data generated in these activities. These understandings are not different from the conceptual goals of DT, so this section discusses the relationship between DPD and DT based on the early research.

\subsection{DMU is the output of design rationale}

The original intention of DPD is to express real physical products. However, taking DMU as an example, the final result becomes "the ideal definition of the product". Due to actual factors such as product deviation, it is impossible to fabricate an ideal product in the real world. Thus, DMU is the output of continuous iteration process of design activities and reflects human design rationale. 


\subsection{DT is the representation of physical product}

The realism holds that the world does not depend on our representation of it. no matter how we describe the world or the description does not exist, the world still exists independently. On the other hand, "model-dependent realism" asserts that all we can know about "reality" consists of networks of world pictures that explain observations by connecting them with rules to concepts defined in models [21]. The realism also suggests that we cannot know "reality-as-it-is-in-itself", but only an "approximation" of it provided by the intermediary of models.

Therefore, in any case, DT cannot be completely reflect the physical product in the real world, but a high fidelity approximation. The DT concept is an available form of representation of the physical product, and it reflects the real world in the virtual space.

\subsection{DT expands the meaning of DPD}

Based on the discussion above, it is suggested that DPD should not be limited to designing phase, but the digital description of all the stages of product life cycle. In fact, the connotation of DPD has been expanding with the development of new technology. When the DMU cannot describe the function and performance of the product, the researchers introduced "FDMU" to describe the functional interface. When the working mode changes from parallel engineering to collaborative engineering, researchers introduced "iDMU" to describe the digital definition of the entire development phase.

In summary, DMU and DT, as two aspects that define ideal products and physical products, can be organically unified, as shown in Figure 5. The merge of DMU and DT reflects the high integration of cyberspace, physical space, and consciousness space. Therefore, DTs will become the "middleware" for in-depth communication between human and machine.

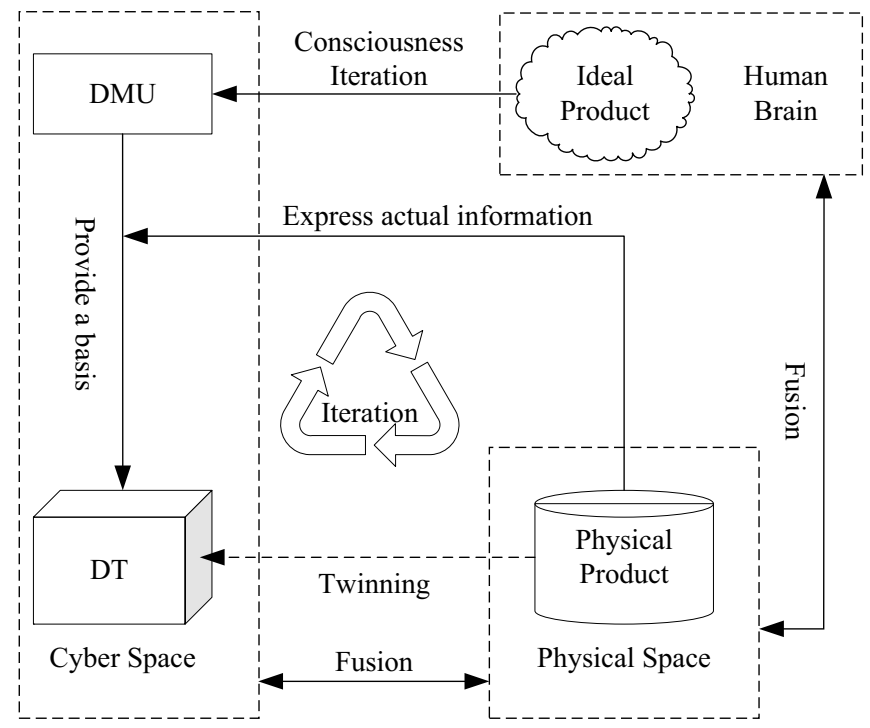

Figure 5. The relationship between DMU \& DT. 


\section{Discussions}

By combing the development trend of DPD, this article summarized the development from DMU to DT, expanded the DPD concept, incorporated DT into DPD, and improved the connotation and connection of DPD in the virtual world and physical world.

With the booming growth of information technologies in the manufacturing industry, remarkable efforts about DT-driven applications are going to challenge the fundamentals of manufacturing systems and operations. Many manufacturing companies nowadays believe that the DT concept could improve the utilization of process knowledge for machining planning.

DTs are the foundation of intelligent manufacturing systems and modeling of DT is an effective way to realize CPS. At present, the research on DT is still at early stage. How to build DTs of physical products requires further research. How to realize the product operation and maintenance based on DTs, and improve the perception feedback, precise control and precise execution of the product life cycle process is the main problem.

\section{References}

[1] Kadiri SE, Kiritsis D. Ontologies in the context of product lifecycle management: state of the art literature review. Int J Prod Res. 2015 Jun;53(18):5657-5668.

[2] Grieves M. Virtually perfect: driving innovative and lean products through product lifecycle management. Florida: Space Coast Press; 2011.

[3] Rosen R, von Wichert G, Lo G, Bettenhausen KD. About the importance of autonomy and digital twins for the future of manufacturing. IFAC-PapersOnLine. $2015 \mathrm{Aug}$;48(3):567-572.

[4] Garbade R, Dolezal WR. DMU@Airbus-evolution of the digital mock-up (DMU) at Airbus to the centre of aircraft development. In: Krause FL, editor. The future of product development. Berlin, Heidelberg: Springer Berlin Heidelberg; 2007. p. 3-12.

[5] Shuichi F, Zoran L, Josip S. FDMU-functional spatial experience beyond DMU? In: Proceedings of the 20th ISPE International Conference on Concurrent Engineering; 2013 Sep 2-6; Melbourne, Australia. Amsterdam: IOS Press; 2013. p. 431-440.

[6] Mas F, Menéndez JL, Oliva M, Ríos J. Collaborative engineering: an Airbus case study. Procedia Eng. 2013 Sep;63:336-345.

[7] Menéndez JL, Mas F, Servan J, Arista R, Ríos J. Implementation of the iDMU for an aerostructure industrialization in Airbus. Procedia Eng. 2013 Sep;63:327-335.

[8] Glaessgen E, Stargel D. The digital twin paradigm for future NASA and U.S. air force vehicles. In: Proceedings of the 53rd AIAA/ASME/ASCE/AHS/ASC Structures, Structural Dynamics and Materials Conference; 2012 Apr 23-26; Honolulu, Hawaii. Reston(VA): AIAA Press; 2012. p. 1818.

[9] Weyer S, Meyer T, Ohmer M, Gorecky D, Zühlke D. Future modeling and simulation of CPS-based factories: an example from the automotive industry. IFAC-PapersOnLine. 2016 Feb;49(31):97-102.

[10] Grieves M, Vickers J. Digital twin: mitigating unpredictable, undesirable emergent behavior in complex systems. In: Kahlen FJ, Shannon F, Anabela A, editors. Transdisciplinary perspectives on complex systems. Cham: Springer International Publishing; 2017. p. 85-113.

[11] Lu Y, Liu C, Wang KIK, Huang H, Xu X. Digital twin-driven smart manufacturing: connotation, reference model, applications and research issues. Robot Cim-Int Manuf. 2020 Feb;61:101837.

[12] Tao F, Cheng J, Qi Q, Zhang M, Zhang H, Sui F. Digital twin-driven product design, manufacturing and service with big data. Int J Adv Manuf Tech. 2017 Mar;94(9-12):3563-3576.

[13] Coronado PDU, Lynn R, Louhichi W, Parto M, Wescoat E, Kurfess T. Part data integration in the Shop Floor Digital Twin: Mobile and cloud technologies to enable a manufacturing execution system. J Manuf Syst. 2018 Jul;48:25-33.

[14] Bao J, Guo D, Li J, Zhang J. The modelling and operations for the digital twin in the context of manufacturing. Enterp Inf Syst. 2018 Oct;13(4):534-556.

[15] Liu J, Zhou H, Liu X, Tian G, Wu M, Cao L, et al. Dynamic evaluation method of machining process planning based on digital twin. IEEE Access. 2019;7:19312-19323. 
[16] Lu Y, Xu X. Cloud-based manufacturing equipment and big data analytics to enable on-demand manufacturing services. Robot Cim-Int Manuf. 2019 Jun;57:92-102.

[17] Leng J, Liu Q, Ye S, Jing J, Wang Y, Zhang C, et al. Digital twin-driven rapid reconfiguration of the automated manufacturing system via an open architecture model. Robot Cim-Int Manuf. 2020 Jun;63:101895.

[18] Zheng P, Sivabalan AS. A generic tri-model-based approach for product-level digital twin development in a smart manufacturing environment. Robot Cim-Int Manuf. 2020 Aug;64:101958.

[19] Jiang H, Qin S, Fu J, Zhang J, Ding G. How to model and implement connections between physical and virtual models for digital twin application. J Manuf Syst. 2020 Jun.

[20] Zhuang C, Gong J, Liu J. Digital twin-based assembly data management and process traceability for complex products. J Manuf Syst. 2020 Jun.

[21] Koonin EV. The logic of chance: the nature and origin of biological evolution. New York: Pearson Education; 2011. 